Pacific Journal of Mathematics

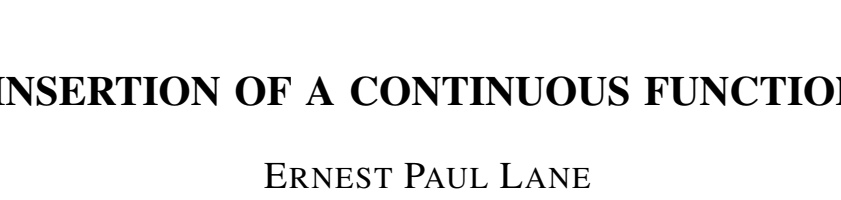




\title{
INSERTION OF A CONTINUOUS FUNCTION
}

\author{
ERnest P. LANe
}

\begin{abstract}
Necessary and sufficient conditions in terms of lower cut sets are given for the insertion of a continuous function between two comparable real valued functions with a certain pair of a general class of properties. The class of properties is defined by being preserved when added to a continuous function and by being possessed by any constant function.
\end{abstract}

A property $P$ defined relative to a real valued function on a topological space is a $C$ property provided any constant function has property $P$ and provided the sum of a function with property $P$ and any continuous function also has property $P$. If $P_{1}$ and $P_{2}$ are $C$ properties, the following terminology is used: (i) A space $X$ has the weak $C$ insertion property for $\left(P_{1}, P_{2}\right)$ if and only if for any functions $g$ and $f$ on $X$ such that $g \leqq f, g$ has property $P_{1}$ and $f$ has property $P_{2}$, then there exists a continuous function $h$ on $X$ such that $g \leqq h \leqq f$. (ii) A space $X$ has the $C$ insertion property for $\left(P_{1}, P_{2}\right)$ if and only if for any functions $g$ and $f$ on $X$ such that $g<f, g$ has property $P_{1}$ and $f$ has property $P_{2}$, then there exists a continuous function $h$ on $X$ such that $g<h<f$. (iii) A space $X$ has the strong $C$ insertion property for $\left(P_{1}, P_{2}\right)$ if and only if for any functions $g$ and $f$ on $X$ such that $g \leqq f$, then there exists a continuous function $h$ on $X$ such that $g \leqq h \leqq f$ and such that if $g(x)<f(x)$ for any $x$ in $X$, then $g(x)<h(x)<f(x)$. If a space $X$ has the weak $C$ insertion property for $\left(P_{1}, P_{2}\right)$, necessary and sufficient conditions in terms of lower cut sets are given for the space to have the $C$ insertion property for $\left(P_{1}, P_{2}\right)$. For a space with the weak $C$ insertion property, a sufficient condition for the space to have the strong $C$ insertion property is given. In certain situations, this condition is necessary. Several insertion theorems, some of which are known, are obtained as corollaries of these two results.

1. Examples of $\boldsymbol{C}$ properties and $\boldsymbol{C}$ insertion. The following are examples of $C$ properties: lower semicontinuous (lsc), upper semicontinuous (usc), normal lsc, normal usc, continuity, measurable, almost continuous (in the sense of Husain [5]), almost continuous (in the sense of Singal and Singal [12]), Baire class one. If $f_{*}$ denotes the lower limit functions of $f$, then $f$ is normal lsc in case $f=\left(f^{*}\right)_{*}$ and $f$ is normal usc in case $f=\left(f_{*}\right)^{*}$; see Dilworth [3] for amplification. Observe that any set of functions that satisfies a given $C$ property contains the set of all continuous functions on the space. 
The following examples of $C$ insertion are known:

(a) A space $X$ has the weak $C$ insertion property for (usc, lsc) if and only if $X$ is normal.

(b) A space $X$ has the $C$ insertion property for (usc, lsc) if and only if $X$ is normal and countably paracompact.

(c) A space $X$ has the strong $C$ insertion property for (usc, lsc) if and only if $X$ is perfectly normal.

(d) A space $X$ has the $C$ insertion property for (usc, continuous) if and only if $X$ is a $c b$-space.

(e) A space $X$ has the $C$ insertion property for (normal usc, continuous) if and only if $X$ is a weak-cb space.

(f) A space $X$ has the weak $C$ insertion property for (normal usc, normal lsc) if and only if $X$ is mildly normal.

(g) A space $X$ has the weak $C$ insertion property for (normal usc, lsc) [respectively, (usc, normal lsc)] if and only if $X$ is almost normal.

(h) A space $X$ has the weak $C$ insertion property for (lsc, usc) if and only if $X$ is extremally disconnected.

Result (a) is due independently to Tong [13] and to Katětov [6]. Example (b) was proved by Katětov [6] and by Dowker [4]. Result (c) is a consequence of Theorem 3.1"', Example 1.2*, and Proposition 2.3 of Michael [11]. Theorem 1 (c) of Mack [9] gives example (d), and (e) is an immediate corollary of Theorem 3.1 (c) of Mack and Johnson [10]. Results (f), (g), and (h) are from Lane [8]. A space is mildly normal in case disjoint regular closed subsets are separated by disjoint open sets, and a space is called almost normal in case disjoint closed sets, at least one of which is regular closed, are separated by disjoint open sets.

If a space has the strong $C$ insertion property for $\left(P_{1}, P_{2}\right)$, then it has the weak $C$ insertion and the $C$ insertion property for $\left(P_{1}, P_{2}\right)$. In order to see when the $C$ insertion property implies the weak $C$ insertion property, a technique used by Dieudonné [2] is employed to prove the following result.

Theorem 1.1. Let $P_{1}$ and $P_{2}$ be $C$ properties and assume that $X$ satisfies the $C$ insertion property for $\left(P_{1}, P_{2}\right)$. If

(a) the transformation $f \rightarrow f /(1+|f|)$ preserves $P_{1}$ and $P_{2}$, and

(b) if $\inf (f, h)$ has property $P_{2}$ and $\sup (g, h)$ has property $P_{1}$ whenever $f$ has property $P_{2}, g$ has property $P_{1}$ and $h$ is any continuous function, then $X$ satisfies the weak $C$ insertion property for $\left(P_{1}, P_{2}\right)$.

Proof. Let $g$ and $f$ be functions on $X$ such that $g \leqq f, g$ has property $P_{1}$ and $f$ has property $P_{2}$. If $G=g /(1+|g|)$ and $F=$ $f /(1+|f|)$, then $G$ has property $P_{1}$ and $F$ has property $P_{2}$ and $-1<G \leqq$ 
$F<1$. If $G_{0}=G-1$ and if $F_{0}=F-1$, then by hypothesis there exists a continuous function $h_{0}$ on $X$ such that $G_{0}<h_{0}<F_{0}$. Let

$$
f_{1}=\inf \left(F+1 / 2, h_{0}+1 / 2\right), \quad g_{1}=\sup \left(G-1 / 2, h_{0}-1 / 2\right) .
$$

Then $g_{1}<f_{1}$ and by hypothesis, $g_{1}$ has property $P_{1}$ and $f_{1}$ has property $P_{2}$. Inductively, let

$$
f_{n}=\inf \left(F+1 / 2^{n}, h_{n-1}+1 / 2^{n}\right), \quad g_{n}=\sup \left(G-1 / 2^{n}, h_{n-1}-1 / 2^{n}\right),
$$

where $h_{n-1}$ is continuous and $g_{n-1}<h_{n-1}<f_{n-1}$. Again by hypothesis there exists a continuous function $h_{n}$ on $X$ such that $g_{n}<h_{n}<f_{n}$. Since $h_{n}<f_{n} \leqq h_{n-1}+1 / 2^{n}$ and since $h_{n}>g_{n} \geqq h_{n-1}-1 / 2^{n}$, then $-1 / 2^{n} \leqq$ $h_{n}-h_{n-1} \leqq 1 / 2^{n}$. Since $\left|h_{n}-h_{n-1}\right| \leqq 1 / 2^{n}$, the sequence $\left\{h_{n}\right\}$ converges uniformly on $X$ by the Cauchy condition. Thus there exists a continuous function $H$ on $X$ such that $\left\{h_{n}\right\}$ converges uniformly on $X$ to $H$. Since

$$
G-1 / 2^{n} \leqq g_{n}<h_{n}<f_{n}<F+1 / 2^{n}
$$

and since $\left\{h_{n}\right\}$ converges to $H$, it follows that $G \leqq H \leqq F$. If $h=$ $H /(1-|H|)$, then $h$ is continuous and $g \leqq h \leqq f$. Thus $X$ satisfies the weak $C$ insertion property for $\left(P_{1}, P_{2}\right)$.

2. $C$ insertion. If $f$ is a real valued function defined on a space $X$ and if

$$
\{x \mid f(x)<r\} \subset A(f, r) \subset\{x \mid f(x) \leqq r\},
$$

for a real number $r$, then $A(f, r)$ is a lower cut set in the domain of $f$ at the level $r$. This definition is due to Brooks [1], where the terminology lower indefinite cut set is used. A sufficient condition in terms of lower cut sets for a space to satisfy the weak $C$ insertion property for arbitrary comparable functions was given by Lane [8]. The main result of this section uses lower cut sets and gives a necessary and sufficient condition for a space that satisfies the weak $C$ insertion property to satisfy the $C$ insertion property.

THEOREM 2.1. Let $X$ be a space that satisfies the weak $C$ insertion property for $C$ properties $P_{1}$ and $P_{2}$. The space $X$ has the $C$ insertion property for $\left(P_{1}, P_{2}\right)$ if and only if there exist lower cut sets $A\left(f-g, 3^{-n+1}\right)$ and there exists a decreasing sequence $\left\{D_{n}\right\}$ of subsets of $X$ with empty intersection and such that for each $n, X-D_{n}$ and $A\left(f-g, 3^{-n+1}\right)$ are completely separated. 
Proof. Assume that $X$ has the weak $C$ insertion property for $C$ properties $P_{1}$ and $P_{2}$. Let $g$ and $f$ be functions such that $g<f, g$ has property $P_{1}$ and $f$ has property $P_{2}$. By hypothesis there exist lower cut sets $A\left(f-g, 3^{-n+1}\right)$ and there exists a sequence $\left\{D_{n}\right\}$ such that $\bigcap_{n=1}^{\infty} D_{n}=$ $\varnothing$ and such that for each $n, X-D_{n}$ and $A\left(f-g, 3^{-n+1}\right)$ are completely separated. Let $k_{n}$ be a continuous function from $X$ into $[0,1]$ such that $k_{n}=0$ on $A\left(f-g, 3^{-n+1}\right)$ and $k_{n}=1$ on $X-D_{n}$. Let a function $k$ on $X$ be defined by

$$
k(x)=1 / 2 \sum_{n=1}^{\infty} 3^{-n} k_{n}(x) .
$$

By the Weierstrass $M$-test the function $k$ is continuous. Since $\bigcap_{n=1}^{\infty} D_{n}=\phi$ and since $k_{n}=1$ on $X-D_{n}$, it follows that $0<k$. Also $2 k<f-g$ : In order to see this, observe first that if $x$ is in $A\left(f-g, 3^{-n+1}\right)$, then $k(x) \leqq 1 / 4\left(3^{-n}\right)$. If $x$ is any point in $X$, then $x \notin A(f-g, 1)$ or for some $n$,

$$
x \in A\left(f-g, 3^{-n+1}\right)-A\left(f-g, 3^{-n}\right)
$$

in the former case $2 k(x)<1$, and in the latter $k(x) \leqq 1 / 4\left(3^{-n}\right) \leqq$ $f(x)-g(x)$. Thus if $f_{1}=f-k$ and if $g_{1}=g+k$, then $g<g_{1}<f_{1}<$ $f$. Since $P_{1}$ and $P_{2}$ are $C$ properties, $g_{1}$ has property $P_{1}$ and $f_{1}$ has property $P_{2}$. Since $X$ has the weak $C$ insertion property for $\left(P_{1}, P_{2}\right)$, there exists a continuous function $h$ on $X$ such that $g_{1} \leqq h \leqq f_{1}$. Since $g<h<f$, it follows that $X$ satisfies the $C$ insertion property for $\left(P_{1}, P_{2}\right)$. (The technique of this proof is from Katětov [6].)

Conversely, let $g$ and $f$ be functions on $X$ such that $g$ has property $P_{1}, f$ has property $P_{2}$ and $g<f$. By hypothesis, there exists a continuous function $h$ on $X$ such that $g<h<f$. Since the constant function 0 has property $P_{1}$, since $f-h$ has property $P_{2}$, and since $X$ has the $C$ insertion property for $\left(P_{1}, P_{2}\right)$, there exists a continuous function $k$ on $X$ such that $0<k<f-h$. Let $A\left(f-g, 3^{-n+1}\right)$ be any lower cut set for $f-g$ and let $D_{n}=\left\{x \mid k(x)<3^{-n+2}\right\}$. Since $k>0$ it follows that $\bigcap_{n=1}^{\infty} D_{n}=$ $\varnothing$. Since

$$
A\left(f-g, 3^{-n+1}\right) \subset\left\{x \mid(f-g)(x) \leqq 3^{-n+1}\right\} \subset\left\{x \mid k(x) \leqq 3^{-n+1}\right\}
$$

and since $\left\{x \mid k(x) \leqq 3^{-n+1}\right\}$ and $\left\{x \mid k(x) \geqq 3^{-n+2}\right\}=X-D_{n}$ are completely separated by $k$, it follows that for each $n, A\left(f-g, 3^{-n+1}\right)$ and $X-D_{n}$ are completely separated.

The first corollary of this theorem is one half of the well known characterization of normal and countably paracompact spaces which is due independently to Dowker [4] and to Katětov [6]. 
COROLlaRY 2.2. If $X$ is normal and countably paracompact, then $X$ has the $C$ insertion property for (usc, lsc).

Proof. Since $X$ is normal, $X$ has the weak $C$ insertion property for (usc, lsc). (See Example (a) of section 1.) Let $g$ and $f$ be functions on $X$ with $g<f, g$ usc and $f$ lsc. For each $n$, let

$$
A\left(f-g, 3^{-n+1}\right)=\left\{x \mid(f-g)(x) \leqq 3^{-n+1}\right\} .
$$

Since $\left\{A\left(f-g, 3^{-n+1}\right)\right\}$ is a decreasing sequence of closed sets and since $X$ is normal and countably paracompact, there exists a decreasing sequence $\left\{D_{n}\right\}$ of open subsets of $X$ such that $D_{n} \supset A\left(f-g, 3^{-n+1}\right)$ and such that $\bigcap_{n=1}^{\infty} D_{n}=\varnothing$. Since $X$ is normal, $A\left(f-g, 3^{-n+1}\right)$ and $X-D_{n}$ are completely separated. Thus by Theorem 2.1 , there exists a continuous function $h$ on $X$ such that $g<h<f$.

For the second corollary, the following result is needed. The proof, which is directly analogous to the proof of Theorem 2 in Dowker's paper [4], is omitted.

LEMMA 2.3. If $X$ is a cb-space and if $\left\{F_{n}\right\}$ is a decreasing sequence of closed sets with empty intersection, then there exists a sequence of regular open sets $\left\{H_{n}\right\}$ such that for each $n, F_{n} \subset H_{n}$ and such that $\bigcap_{n=1}^{\infty} \bar{H}_{n}=\varnothing$.

COROllary 2.4. A space $X$ has the $C$ insertion property for (usc, normal lsc) [respectively (normal usc, lsc)] if $X$ is almost normal and a cb-space.

Proof. The proof is given for (usc, normal lsc). Since $X$ is almost normal, $X$ has the weak $C$ insertion property for (usc, normal lsc) (Lane [8]). If $g$ and $f$ are functions on $X$ such that $g<f, g$ is usc, and $f$ is normal lsc, and if

$$
A\left(f-g, 3^{-n+1}\right)=\left\{x \mid(f-g)(x) \leqq 3^{-n+1}\right\},
$$

then $\left\{A\left(f-g, 3^{-n+1}\right)\right\}$ is a decreasing sequence of closed lower cut sets with empty intersection. Since $X$ is a $c b$-space, it follows from Lemma 2.3 that there exists a decreasing sequence $\left\{H_{n}\right\}$ of regular open subsets of $X$ such that $A\left(f-g, 3^{-n+1}\right)$ is contained in $H_{n}$ and such that $\bigcap_{n=1}^{\infty} \bar{H}_{n}=$ $\varnothing$. Since $X$ is almost normal, $A\left(f-g, 3^{-n+1}\right)$ and the regular closed set $X-H_{n}$ are completely separated. Thus $A\left(f-g, 3^{-n+1}\right)$ and $X-\bar{H}_{n}$ are completely separated. By Theorem 2.1, there exists a continuous function $h$ on $X$ such that $g<h<f$.

The proof of the next corollary is omitted since the technique is the same as in the preceding two corollaries. The main result needed is that 
an extremally disconnected space has the weak $C$ insertion property for (lsc, usc) (Lane [8]).

Corollary 2.5. A space $X$ has the $C$ insertion property for (lsc, usc) if $X$ is extremally disconnected and if for any decreasing sequence $\left\{G_{n}\right\}$ of open subsets of $X$ with empty intersection there exists a decreasing sequence $\left\{F_{n}\right\}$ of closed subsets of $X$ with vacuous intersection such that $F_{n} \supset G_{n}$ for each $n$.

In each of the preceding three corollaries, the sufficient condition for $C$ insertion is also a necessary condition.

\section{Strong $C$ insertion.}

THEOREM 3.1. Let $P_{1}$ and $P_{2}$ be $C$ properties and consider the following condition:

$(\alpha)$ If $g$ and $f$ are functions on $X$ such that $g \leqq f, g$ satisfies property $P_{1}$ and $f$ satisfies property $P_{2}$, then there exists a sequence $\left\{A\left(f-g, 2^{-n}\right)\right\}$ of lower cut sets for $f-g$ and there exists a sequence $\left\{F_{n}\right\}$ of subsets of $X$ such that

(i) $\{x \mid(f-g)(x)>0\}=\bigcup_{n=1}^{\infty} F_{n}$, and

(ii) for each $n$ the sets $A\left(f-g, 2^{-n}\right)$ and $F_{n}$ are completely separated.

If $X$ satisfies the weak $C$ insertion property for $\left(P_{1}, P_{2}\right)$ and if $X$ satisfies condition $(\alpha)$, then $X$ satisfies the strong $C$ insertion property for $\left(P_{1}, P_{2}\right)$. Conversely, if $X$ satisfies the strong $C$ insertion property for $\left(P_{1}, P_{2}\right)$ and if $\hat{j}-g$ satisfies property $P_{1}$, then $X$ satisfies condition $(\alpha)$.

Proof. Suppose that there is a sequence $\left\{A\left(f-g, 2^{-n}\right)\right\}$ of lower cut sets for $f-g$ and suppose there is a sequence $\left\{F_{n}\right\}$ of subsets of $X$ such that

$$
\{x \mid(f-g)(x)>0\}=\bigcup_{n=1}^{\infty} F_{n}
$$

and such that for each $n$ there exists a continuous function $k_{n}$ from $X$ into $\left[0,2^{-n}\right]$ with $k_{n}=2^{-n}$ on $F_{n}$ and $k_{n}=0$ on $A\left(f-g, 2^{-n}\right)$. The function $k$ from $X$ into $[0,1 / 4]$ which is defined by

$$
k(x)=1 / 2 \sum_{n=1}^{\infty} k_{n}(x)
$$

is continuous, (1) $k^{-1}(0)=\{x \mid(f-g)(x)=0\}$ and (2) if $(f-g)(x)>0$ then $k(x)<(f-g)(x)$ : In order to verify (1), observe that if $(f-g)(x)=$ 
0 , then $x \in A\left(f-g, 2^{-n}\right)$ for each $n$ and hence $k_{n}(x)=0$ for each $n$. Thus $k(x)=0$. Conversely, if $(f-g)(x)>0$, then there exists an $n$ such that $x \in F_{n}$ and hence $k_{n}(x)=2^{-n}$. Thus $k(x) \neq 0$ and this verifies (1). Next, in order to establish (2), note that

$$
\{x \mid(f-g)(x)=0\}=\bigcap_{n=1}^{\infty} A\left(f-g, 2^{-n}\right)
$$

and that $\left\{A\left(f-g, 2^{-n}\right)\right\}$ is a decreasing sequence. Thus if $(f-g)(x)>0$ then either $x \notin A(f-g, 1 / 2)$ or there exists a smallest $n$ such that $x \notin A\left(f-g, 2^{-n}\right)$ and $x \in A\left(f-g, 2^{-j}\right)$ for $j=1, \cdots, n-1$. In the former case,

$$
k(x)=1 / 2 \sum_{n=1}^{\infty} k_{n}(x) \leqq 1 / 4 \sum_{n=1}^{\infty} 2^{-n}<1 / 2 \leqq(f-g)(x),
$$

and in the latter,

$$
k(x)=1 / 4 \sum_{j=n}^{\infty} k_{\jmath}(x) \leqq 1 / 4 \sum_{j=n}^{\infty} 2^{-\jmath}<2^{-n} \leqq(f-g)(x) .
$$

Thus $0 \leqq k \leqq f-g$ and if $(f-g)(x)>0$ then $(f-g)(x)>k(x)>0$. Let $g_{1}=g+(1 / 4) k$ and let $f_{1}=f-(1 / 4) k$. Then $g \leqq g_{1} \leqq f_{1} \leqq f$ and if $g(x)<f(x)$ then

$$
g(x)<g_{1}(x)<f_{1}(x)<f(x) .
$$

Since $P_{1}$ and $P_{2}$ are $C$ properties, $g_{1}$ has property $P_{1}$ and $f_{1}$ has property $P_{2}$. Since $X$ has the weak $C$ insertion property for $\left(P_{1}, P_{2}\right)$ by hypothesis, there exists a continuous function $h$ on $X$ such that $g_{1} \leqq h \leqq f_{1}$. Thus $g \leqq h \leqq f$ and if $g(x)<f(x)$ then $g(x)<h(x)<f(x)$. Therefore $X$ has the strong $C$ insertion property for $\left(P_{1}, P_{2}\right)$.

Conversely, assume that $X$ satisfies the strong $C$ insertion property for $\left(P_{1}, P_{2}\right)$ and assume that $g$ and $f$ are functions on $X$ such that $g \leqq f$ and such that $f-g$ satisfies property $P_{1}$. There exists a continuous function $h$ on $X$ such that $0 \leqq h \leqq f-g$ and such that if $(f-g)(x)>0$ then

$$
(f-g)(x)>h(x)>0 .
$$

For each $n$, let

$$
\begin{aligned}
& A\left(f-g, 2^{-n}\right)=\left\{x \mid(f-g)(x) \leqq 2^{-n}\right\} \\
& F_{n}=\left\{x \mid h(x) \geqq 2^{-n+1}\right\}, \text { and } \\
& k_{n}=\sup \left\{\inf \left\{h, 2^{-n+1}\right\}, 2^{-n}\right\}-2^{-n} .
\end{aligned}
$$


Then $\{x \mid(f-g)(x)>0\}=\bigcup_{n=1}^{\infty} F_{n}$ and $k_{n}$ is a continuous function from $X$ into $\left[0,2^{-n}\right]$ which completely separates $F_{n}$ and $A\left(f-g, 2^{-n}\right)$.

The first corollary of this theorem is the result of Michael [11] which was listed above.

COROllary 3.2. A space $X$ satisfies the strong $C$ insertion property for (usc, lsc) if and only if $X$ is perfectly normal.

Proof. The proof of the sufficiency of the insertion property for perfect normality is omitted. To see how the necessity of the condition follows from Theorem 3.1, suppose that $X$ is perfectly normal and let $f$ and $g$ be functions on $X$ such that $g \leqq f, g$ is usc and $f$ is lsc. Since $X$ is normal, $X$ satisfies the weak $C$ insertion property for $\left(P_{1}, P_{2}\right)$. Since $f-g$ is lsc, the lower cut sets for $f-g$ defined by

$$
A\left(f-g, 2^{-n}\right)=\left\{x \mid(f-g)(x) \leqq 2^{-n}\right\}
$$

are closed sets. Since $X$ is perfectly normal, there exists a sequence $\left\{F_{n}\right\}$ of closed subsets of $X$ such that

$$
\{x \mid(f-g)(x)>0\}=\bigcup_{n=1}^{\infty} F_{n}
$$

and such that for each $n, F_{n}$ and $A\left(f-g, 2^{-n}\right)$ are completely separated. Hence by Theorem $3.1 X$ satisfies the strong $C$ insertion property for (usc, lsc).

Corollary 3.3. A space $X$ satisfies the strong $C$ insertion property for (usc, normal lsc) [respectively, (normal usc, lsc)] if and only if $X$ is almost normal and if each closed subset of $X$ is an intersection of a sequence of regular open sets.

Proof. Assume that $X$ is almost normal and assume that each closed subset of $X$ is an intersection of a sequence of regular open sets. Since $X$ is almost normal, $X$ satisfies the weak $C$ insertion property for (usc, normal lsc) as noted in Example (g) above. Let $g$ and $f$ be functions on $X$ such that $g \leqq f$, and such that $g$ is usc and $f$ is normal lsc. Since $f-g$ is lsc, the lower cut set for $f-g$ defined by

$$
A\left(f-g, 2^{-n}\right)=\left\{x \mid(f-g)(x) \leqq 2^{-n}\right\}
$$

is a closed set for each $n$. By hypothesis, each open subset of $X$ is a union of a sequence of regular closed sets; thus it is possible to choose a 
sequence of regular closed sets $\left\{F_{n}\right\}$ such that for each $n, F_{n}$ and $A\left(f-g, 2^{-n}\right)$ are disjoint and such that

$$
\{x \mid(f-g)(x)>0\}=\bigcup_{n=1}^{\infty} F_{n} .
$$

Since $X$ is almost normal, the regular closed set $F_{n}$ is completely separated from the closed set $A\left(f-g, 2^{-n}\right)$ by Theorem 3.5 of Lane [7]. Thus it follows from Theorem 3.1 that $X$ satisfies the strong $C$ insertion property for (usc, normal lsc).

If $X$ satisfies the strong $C$ insertion property for (usc, normal lsc) then $X$ satisfies the weak $C$ insertion property for (usc, normal lsc) and by Example (g) above, $X$ is almost normal. In order to see that each closed subset of $X$ is an intersection of a sequence of regular open sets, let $F$ be a closed subset of $X$. Let $f=1$ on $X$, and let $g=1$ on $F$ and $g=0$ on $X-F$. Since $g \leqq f$, and since $g$ is usc and $f$ is normal lsc, there is a continuous function $h$ on $X$ such that $g \leqq h \leqq f$ and such that if $x \in X-F$ then $0<h(x)<1$. If $G_{n}$ is the interior of the closed set $\{x \mid h(x) \geqq 1-1 / n\}$, then $\left\{G_{n}\right\}$ is a sequence of regular open sets whose intersection is $F$.

The proof for the (normal usc, lsc) is analogous and is omitted.

The proofs of the following two results are essentially the same as the preceding corollary and are not given. Note that in both cases, the space trivially satisfies the weak $C$ insertion property.

Corollary 3.3. An almost normal space $X$ satisfies the strong $C$ insertion property for (usc, continuous) [respectively, (continuous, lsc)] if and only if each closed subset of $X$ is an intersection of a sequence of regular open sets.

COROLlaRY 3.4. If $X$ is almost normal and if each closed subset of $X$ is an intersection of a sequence of regular open sets, then $X$ satisfies the strong $C$ insertion property for (normal usc, continuous) [respectively, (continuous, normal lsc)].

\section{REFERENCES}

1. F. Brooks, Indefinite cut sets for real functions, Amer. Math. Monthly, 78 (1971), 1007-1010.

2. J. Dieudonné, Une généralisation des espaces compacts, Journal de Math. Pures et Appliqués, 23 (1944), 65-76.

3. R. P. Dilworth, The normal completion of the lattice of continuous functions, Trans. Amer. Math. Soc., 68 (1950), 427-438.

4. C. H. Dowker, On countably paracompact spaces, Canad. J. Math., 3 (1951), 219-224.

5. T. Husain, Almost continuous mappings, Prace Mat. Series 1, 10 (1965), 1-7.

6. M. Katětov, On real-valued functions in topological spaces, Fund. Math., 38 (1951), 85-91. 
7. E. Lane, Insertion of continuous functions, Glasnik Mat. Series III, 6 (25) (1971), 165-171.

8. - A sufficient condition for the insertion of a continuous function, Proc. Amer. Math. Soc., 49 (1975), 90-94.

9. J. E. Mack, On a class of countably paracompact spaces, Proc. Amer. Math. Soc., 16 (1965), 467-472.

10. J. E. Mack and D. G. Johnson, The Dedekind completion of $C(X)$, Pacific J. Math., 20 (1967), 231-243.

11. E. Michael, Continuous selections I, Ann. of Math., 63 (1956), 361-382.

12. M. K. Singal and A. R. Singal, Almost-continuous mappings, Yokohama Math. J., 16 (1968), $63-73$.

13. H. Tong, Some characterizations of normal and perfectly normal spaces, Duke Math. J., 19 (1952), 289-292.

Received March 22, 1976.

Appalachian State University 



\section{Pacific Journal of Mathematics}

\section{Vol. 66, No. $1 \quad$ November, 1976}

Helen Elizabeth. Adams, Factorization-prime ideals in integral domains ............ Patrick Robert Ahern and Robert Bruce Schneider, The boundary behavior of Henkin's kernel.

Daniel D. Anderson, Jacob R. Matijevic and Warren Douglas Nichols, The Krull

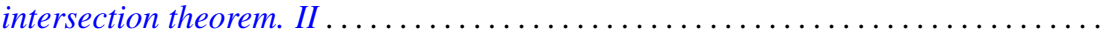

Efraim Pacillas Armendariz, On semiprime P.I.-algebras over commutative regular

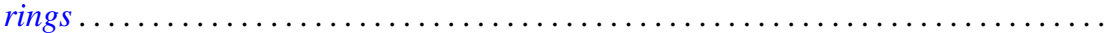

Robert H. Bird and Charles John Parry, Integral bases for bicyclic biquadratic fields

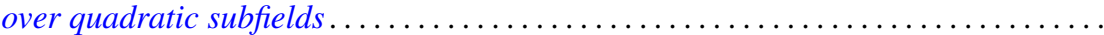

Tae Ho Choe and Young Hee Hong, Extensions of completely regular ordered

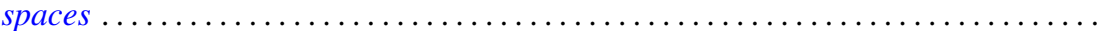

John Dauns, Generalized monoform and quasi injective modules ...............

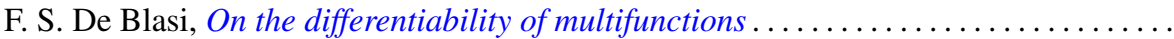

Paul M. Eakin, Jr. and Avinash Madhav Sathaye, R-endomorphisms of $R[[X]]$ are

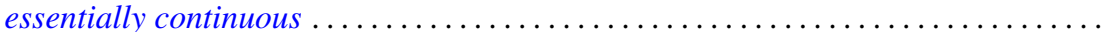

Larry Quin Eifler, Open mapping theorems for probability measures on metric

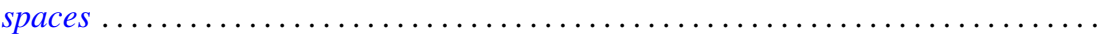

Garret J. Etgen and James Pawlowski, Oscillation criteria for second order self adjoint

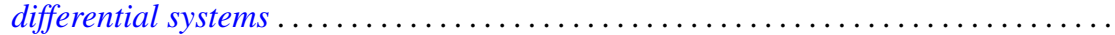

Ronald Fintushel, Local $S^{1}$ actions on 3-manifolds .

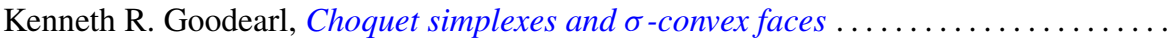

John R. Graef, Some nonoscillation criteria for higher order nonlinear differential

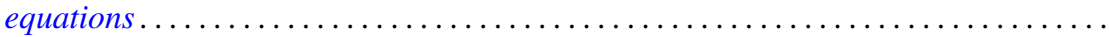

Charles Henry Heiberg, Norms of powers of absolutely convergent Fourier series: an example.

Les Andrew Karlovitz, Existence of fixed points of nonexpansive mappings in a space

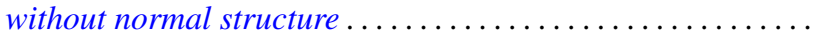

Gangaram S. Ladde, Systems of functional differential inequalities and functional

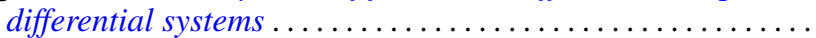

Joseph Michael Lambert, Conditions for simultaneous approximation and interpolation with norm preservation in $C[a, b]$.

Ernest Paul Lane, Insertion of a continuous function.

Robert F. Lax, Weierstrass points of products of Riemann surfaces .

Dan McCord, An estimate of the Nielsen number and an example concerning the Lefschetz fixed point theorem...

Paul Milnes and John Sydney Pym, Counterexample in the theory of continuous functions on topological groups...

Peter Johanna I. M. De Paepe, Homomorphism spaces of algebras of holomorphic functions

Judith Ann Palagallo, A representation of additive functionals on $L^{p}$-spaces,

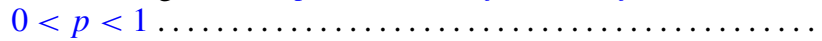

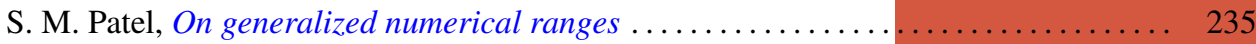

Thomas Thornton Read, A limit-point criterion for expressions with oscillatory

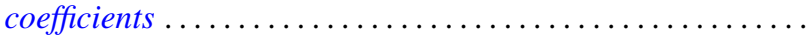

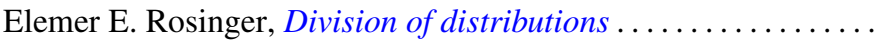

Peter S. Shoenfeld, Highly proximal and generalized almost finite

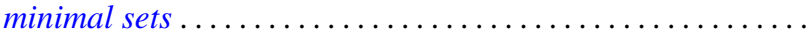

R. Sirois-Dumais and Stephen Willard, Quotient-universal sequential spaces

Robert Charles Thompson, Convex and concave functions of singular values of matrix sums....

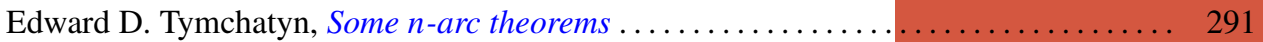

